\section{Changes in auditory flutter fusion frequency during prolonged visual deprivation*}

\author{
C. H. PANGMAN and JOHN P. ZUBEK \\ University of Manitoba, Winnipeg, Manitoba, Canada
}

The auditory flutter fusion frequency of 18 Ss was determined at intervals of $0,1 / 2,1,2,3,5$, and 7 days of vicual deprivation (darkness). Relative to control Ss, a progressive improvement in performance as a function of duration was observed, a functional relationship which was best represented by a fourth-order polynomial. These results were similar to those obtained in an earlier experiment in which a tactual fusion task was employed, a measure analogous to auditory flutter fusion.

Recently, Milstein and Zubek (1971) conducted a series of experiments in which various measures of cutaneous sensitivity were administered at periodic intervals during 1 week of visual deprivation (darkness). Relative to control Ss, the results revealed no significant differences in performance, at any temporal period, on measures of absolute pressure sensitivity, the two-point limen, and pain sensitivity. On the other hand, the results on a tactual fusion task, a measure analogous to the visual CFF, revealed a progressive improvement in performance as a function of duration of visual deprivation, a facilitatory effect which was already present, to a statistically significant degree, at the first test period-12 h. (The fourth-order polynomial was found to be the curve of best fit.)

In an attempt to account for these differential results, the authors suggested that the temporal discriminatory nature of the tactual fusion task, a stimulus characteristic not present in the other three cutaneous measures, might be a factor. Some support for the importance of this distinction has been provided by Duda and Zubek (1965), who, in attempting to explain some differential auditory effects occurring after a week of visual deprivation (viz, an improved performance on an auditory flutter fusion task but no change in the absolute threshold of hearing for pure tones), reported that "the auditory facilitatory effects may apply only to sensory tests involving a temporal discrimination."

If, as has been hypothesized, the temporal discriminatory nature of the tactual fusion task is an important

* This research was supported by the Defence Research Board, Canada (Grant 9425-08) and by the National Research Council, Canada (Grant AP A-290) The authors wish to expres their appreciation to L. Bayer, M. Brosziewsi. and $T$. Gutkin for research assistance. factor in producing these results, one might expect a similar pattern of improvement on other analogous types of tasks, also involving temporal resolutions, e.g., on a measure of auditory flutter fusion. In order to test this hypothesis, an experiment was designed in which this particular auditory measure was administered at intervals of $0,1 / 2,1,2,3,5$, and 7 days of visual deprivation-test durations similar to those employed in the Milstein and Zubek study. Such an experiment may provide some useful information as to the specificity or generality of some of the sensory facilitatory phenomena which have been reported (see Zubek, 1969, for a review of this general topic). For example, is the progressive improvement on the tactual fusion task specific to the use of a cutaneous measure, employing intermittent stimulation, or can this temporal phenomenon also be observed on a similar type of noncutaneous measure?

\section{METHOD}

Subjects

The Ss were 36 male university students, ranging in age from 17 to 25 years (mean age $=18.9$ years), all of whom volunteered to spend a week in darkness. They were assigned randomly to an experimental and a control group, each containing $18 \mathrm{Ss}$. Both groups were paid for their participation.

The experimental Ss, each wearing a black mask over both eyes, were placed in groups of two in a furnished air-conditioned room $(4.6 \times 3.0 \mathrm{~m})$ for a prescribed period of 1 week. Apart from the exposure to constant darkness, their environment was normal. They were free to move about the room, to converse with each other, and to listen to a radio during the day and evening. They were confined to the room for the entire week, except for periodic visits to the auditory test chamber and a washroom. At these times they were accompanied by $\mathrm{E}$.
Test Procedure

The auditory measurements were taken in an acoustical chamber (Model 1405 ACT, Industrial Acoustical Corp.) at intervals of $0,1 / 2$, $1,2,3,5$, and 7 days of visual deprivation. A practice session was given 1 day prior to the experiment. With the exception of the 12-h test, given in the evening, all of the measurements were taken in the morning between $8: 45$ a.m. and 10:00 a.m. Each individual $S$, however, had his specific test time which did not vary from day to day by more than 5 min.

The 18 control Ss came to the laboratory eight times during the experimental period. They were tested in the same acoustical chamber and at the same time of the day as the experimentals. After completion of the tests, they left the laboratory and returned the next day. 1 Both groups of Ss were run concurrently.

Auditory sensitivity was determined by a fusion or flutter method. This measure, which is considered to be an auditory analogue of visual flicker (Miller \& Taylor, 1948), involves the production of an interrupted white noise whose frequency of interruption can be systematically increased until $S$ reports a constant sensation of noise. The frequency at which this constant sensation occurs is usually referred to as the auditory flutter fusion frequency (AFF). The interrupted white noise, at an on-off ratio of 0.90 and an intensity of approximately $70 \mathrm{~dB}$ re $0.0002 \mathrm{dynes} / \mathrm{cm}^{2}$, was presented binaurally by a noise a udiometer (Grason-Stadler, Model 830-52) and a pair of earphones (Grason-Stadler, Model D30). The method of limits, with descending series only, was used to determine the fusion threshold, since it has been shown that "a minimal flutter stands out as a figure in a ground of repeated continuous noise bursts more distinctly than the reverse [Symmes, Chapman, \& Halstead, 1955]." Four practice trials, followed by two sets of three experimental trials, each session being separated by a 1-min rest period, were administered on each test day. The mean of six trials was taken as the S's auditory flutter fusion threshold.

Previous research at this laboratory has indicated that in order to reduce intra-S variability in performance on this particular task, practice periods are required at each test day. The employment of this procedure, together with the use of rest periods and the descending method of limits provided for a consistent set of responses from session to session.

RESULTS

Figure 1 summarizes the results on 


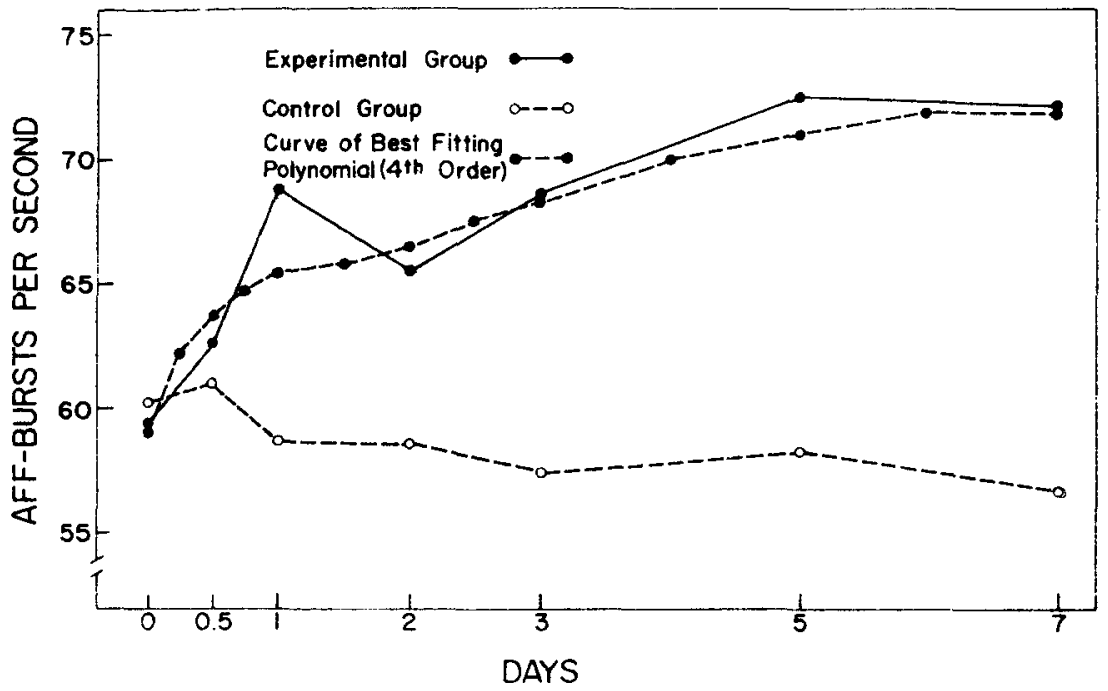

Fig. 1. Temporal changes in auditory flutter fusion frequency (AFF) in two groups of Ss before and during a week of visual deprivation or of a control condition. This figure also shows the polynomial (fourth-order) which best fits the data obtained from the experimental group of Ss.

the AFF. It can be seen that the experimental $S s$, relative to the controls, show an improvement in performance of a negatively accelerated nature as a function of duration of visual deprivation. An analysis of variance (mixed design for repeated measures, Myers, 1966) performed on this data revealed a significant change over days $(F=2.23$; $\mathrm{p}<.05$ ) and a significant interaction effect $(F=5.78 ; p<.01)$. Since the interaction effect was significant, a series of individual two-tailed $t$ tests for independent groups was performed, comparing the relative performance of the experimental and control Ss at the various temporal periods. This analysis revealed that only the increased auditory performance observed during the second half of the experimental period, i.e., Days 3, 5, and 7, was statistically significant (ps $<.05$ ).

An examination of Fig. 1 appears to indicate that the performance on Day 1 is considerably better than that on Day 2. This result, however, is due almost entirely to one $S$ whose score on Day 1 was abnormally high. His elimination from the sample would reduce the Day 1 mean score from 68.9 to 65.3. (This mean is consistent with the data obtained by the best-fit polynomial.)

Although the control group showed a trend toward progressively lower AFF scores over the 1 -week period, no significant difference was obtained between Day 0 and Day 7 .

In order to determine the nature of the functional relationship between the changes in auditory performance and duration of deprivation, a weighted least-square solution was used in computing the regression equation for the polynomials best fitting the AFF scores of the experimental Ss (see Halasz, 1968). Attention was paid to the reliability of the data at each time period by weighting the mean score for each time period by the standard deviation of the scores. (This procedure is functionally equivalent to the reciprocal of the variance, used by Halasz, but is somewhat more convenient.) In addition to giving the evaluated points for each test session, an interpolated value was given between each experimental or actual data point. Both the polynomials and the root mean square (RMS) error were obtained for Orders 1-10, with the order polynomial having the smallest RMS error being accepted as the best representation of the data. The results of this analysis indicated that the fourth-order polynomial (see Fig. 1) was the curve of best fit (RMS =0.089).

\section{DISCUSSION}

A comparison of the present results on auditory fusion with those obtained by Milstein and Zubek (1971) on tactual fusion indicates a similar developmental pattern. Both measures show a progressive improvement in performance, as a function of duration, with some fifth day. Furthermore, in both cases, the functional relationship was best represented by a fourth-order polynomial. In view of this similarity, it would appear that when sensory measures of a temporal discriminatory nature are administered periodically during a prolonged period of visual indication of a leveling off after the deprivation, a progressive improvement in performance seems to occur, a phenomenon similar to that of stimulus-seeking behavior which is known to increase with time in sensory isolation(see Jones, 1969 , for a review of this literature). However, the question still remains as to why sensory measures, employing nonflickering stimuli, do not show the same temporal pattern of results during prolonged deprivation. Only further research can provide a satisfactory answer.

These intersensory facilitatory effects, observed on both the tactual fusion and auditory fusion tasks, are believed to be mediated by the reticular activating system (RAS). First, this neural system receives afferent impulses from various sensory sources via collaterals of ascending tract fibers and transmits them diffusely to the cerebral cortex. Thus a mechanism for intersensory effects is present. Second, it has been suggested by Lindsley (1961) that the RAS may act "as a sort of homeostat regulating or adjusting input-output relations. ... Its changes and adjustments depend upon the ebb and flow of activity in the afferent or efferent systems and when these are restricted, compensatory adjustments are made." One of these compensatory adjustments may be a facilitation of performance on certain sensory measures, particularly those of a temporal discriminatory nature.

With regard to future research in this area, it would be important to determine if a prolonged period of auditory deprivation can also produce a progressive improvement in performance as a functon of duration, if such measures as the visual CFF and tactual fusion, both involving an intermittent form of stimulation, were to be employed. This type of experiment should indicate whether the functional relationship that we have obtained in this and in our earlier study is specific to the deprivation of the visual modality or whether it is of a more general nature operating across several modalities. Since only sensory measures of an analogous nature would be employed, theprobability of discovering any general functional relationship, if it exists, should be considerably enhanced.

\section{REFERENCES}

DUDA, P., \& ZUBEK, J. P. Auditory sensitivity after prolonged visual deprivation. Psychonomic Science, 1965, 3. 359-360.

HALASZ, M. F. A behavioral evoked response: Probing the stability of delayed conditioned approach with impulse-like changes of reinforcement schedule. Canadian Journal of Psychology, 1968, 22, 229-243.

JONES, A. Stimulus-seeking behavior. In J. P. Zubek (Ed.), Sensory deprivation: 
Fifteen years of research. New York: Appleton-Century-Crofts, 1969. Pp. 167-206

LINDSLEY, D. B. Common factors in sensory deprivation, sensory distortion. and sensory overload. In P. Solomon et al (Eds.), Sensory deprivation. Cambridge: Harvard University - Press, 1961. Pp. 174-194.

MILLER, G. A., \& TAYLOR, W. G. The perception of repeated bursts of noise. Journal of the Acoustical Society of America, 1948, 20, 171-182.

MILSTEIN, S, L. \& ZUBEK, J. P. Temporal changes in cutaneous sensitivity during prolonged visual deprivation. Canadian Journal of Psychology, 1971, 25,
336-348.

MYERS, J. L. Fundamentals of experimental design. Boston: Allyn \& Bacon, 1966.

SYMMES, D., CHAPMAN, L. F., \& HALSTEAD, W. C. The fusion of intermittent white noise. Journal of the Acoustical Society of America, 1955, 27. 470-473.

ZUBEK, J. P. Sensory and perceptual-motor processes. In J. P. Zubek (Ed.), Sensory deprivation: Fifteen years of research. 1969. Pp. 207-253.

NOTE

1. Ideally, a 1-week confinement control group should have been employed for comparative purposes. Such a group was not employed for financial reasons and also because some exploratory research, conducted several years ago, indicated that a week of confinement alone, in a windowless room, produced no significant effect on the $A F F$ and on various cutaneous measures. The $\mathrm{Ss}$ in this exploratory study lived under the same conditions as did the present experimental Ss. However, they wore no black mask and their room was illuminated during the daytime and evening hours.

(Accepted for publication September 17, 1971.)

\section{ERRATUM}

HENDERSON, DAVID C., The relationships among time, distance, and intensity as determinants of motion discrimination. Perception \& Ps ychophysics, 1971,10 , 313-320.-The citation footnote on P. 313 should have included the following sentence: This investigation was supported by PHS Research Grants 5 R01 EYO0391 and 5R01 EY00375 from the National Eye Institute, Research Grant GB 5947 from the National Science Found a $t$ ion, and by Contract N00014-67 A-0108-0009 between Columbia University and the Office of Naval Research. 